\title{
Risk Factors and Characterization of Post-COVID-19 Syndrome in Jordan
}

Mohamad-Said Almasri; Rashed Alqaisi; Mohammad Al-Shagahin; Waqar Al-Kubaisy; Ahmad Aljarajreh; Hani Al-Shagahin

Faculty of Medicine, Mutah University, Karak, Jordan

Corresponding Author:

Mohamad-Said Almasri

Faculty of Medicine

Mutah University

Mutah Street

Karak, 210053

Jordan

Phone: 962790720919

Email: mihammedsaidalmasri@gmail.com

\section{Abstract}

Background: There is controversial information about the sequelae of COVID-19 after recovery, or post-COVID-19 syndrome (PCS). Despite the considerable number of studies on COVID-19, proportionally, there is a scarcity of literature addressing PCS, particularly the risk factors causing this syndrome. Determining the prevalence, most common manifestations of PCS, and the possible related risk factors is an important issue.

Objective: To fill these gaps, the aim of this study was to detect the prevalence and risk factors for the development of PCS, and to identify the symptoms and their relation to the sociodemographic and medical characteristics of patients who survived COVID-19 after more than 3 months from onset of illness throughout Jordan.

Methods: A cross-sectional, online questionnaire-based study was conducted. This questionnaire was posted to the association of "My experience with COVID-19" in Jordan. Sociodemographic and COVID-19 illness information was collected from 657 patients who had recovered from COVID-19 at least 3 months after the illness started.

Results: The PCS prevalence was 71.9\%, including patients who experienced at least one PCS symptom. The most common symptoms included dyspnea, fatigue, taste and smell impairment, cough, and depression. Six factors were found to significantly increase the risk of PCS: being female (odds ratio [OR] 2.06, 95\% CI 1.409-2.856), aged $\geq 30$ years (OR 1.64, 95\% CI 1.16-2.33), diabetes mellitus (OR 2.978, 95\% CI 1.08-8.21), hypertension (OR 2.22, 95\% CI 1.118-4.423), respiratory disease (OR 2.33, 95\% CI 1.21-4.501), and neuropsychological disturbance during illness (OR 3.79, 95\% CI 2.574-5.573). These patients also showed a significantly higher rate of PCS than their counter groups. Therefore, females, aged $\geq 30$ years, comorbidity, and neuropsychological disturbance during illness are considered to be risk factors for PCS.

Conclusions: The PCS prevalence is high in Jordan, particularly among certain populations such as females; aged $\geq 30$ years; those with a neuropsychological disturbance during illness; and having a comorbidity such as diabetes, hypertension, and respiratory diseases, which were associated with a significantly higher risk for the development of PCS manifestations. In other words, these populations should be considered as a risk group for PCS occurrence. Therefore, COVID-19 infection treatment should not only be administered during the acute episode but should continue for several months after recovery of the patient. In addition, the PCS period will require further scientific study and investigation along with early interventions, including rehabilitation. Therefore, we now have to start the steps in preparing for this unavoidable problem to improve the health care system and enhance the management of patients during the PCS period. Psychological and medical support is highly recommended during and after a COVID-19 episode, particularly for the high-risk groups.

(iproc 2022;8(1):e36563) doi: $\underline{10.2196 / 36563}$

\section{KEYWORDS}

post-COVID-19 syndrome; COVID-19; postacute sequelae of SARS-CoV-2 infection; long haul; long COVID-19; chronic COVID-19 syndrome; epidemiology; risk factors; symptoms; Jordan 
Edited by Y Khader; this is a non-peer-reviewed article. Submitted 17.01.22; accepted 19.01.22; published 07.02.22.

Please cite as:

Almasri MS, Alqaisi R, Al-Shagahin M, Al-Kubaisy W, Aljarajreh A, Al-Shagahin H

Risk Factors and Characterization of Post-COVID-19 Syndrome in Jordan

iproc 2022;8(1):e36563

URL: https://www.iproc.org/2022/1/e36563

doi: $\underline{10.2196 / 36563}$

PMID:

(CMohamad-Said Almasri, Rashed Alqaisi, Mohammad Al-Shagahin, Waqar Al-Kubaisy, Ahmad Aljarajreh, Hani Al-Shagahin. Originally published in Iproceedings (https://www.iproc.org), 07.02.2022. This is an open-access article distributed under the terms of the Creative Commons Attribution License (https://creativecommons.org/licenses/by/4.0/), which permits unrestricted use, distribution, and reproduction in any medium, provided the original work, first published in Iproceedings, is properly cited. The complete bibliographic information, a link to the original publication on https://www.iproc.org/, as well as this copyright and license information must be included. 